\title{
Critical review of directional neutron survey meters
}

\author{
Matthew J. I. Balmer ${ }^{\mathrm{a}, *}$, Kelum A. A. Gamage ${ }^{\mathrm{a}}$, Graeme C. Taylor ${ }^{\mathrm{b}}$ \\ ${ }^{a}$ Department of Engineering, Lancaster University, LA1 4YR, UK \\ ${ }^{b}$ Neutron Metrology Group, National Physical Laboratory, Teddington, TW11 OLW, UK
}

\begin{abstract}
Having been overlooked for many years, research is now starting to take into account the directional distribution of the neutron work place field. The impact of not taking this into account has led to overly conservative estimates of dose in neutron workplace fields. This paper provides a critical review of this existing research into directional survey meters which could improve these estimates of dose. Instruments which could be adapted for use as directional neutron survey meters are also considered within this review. Using Monte-Carlo techniques, two of the most promising existing designs are evaluated; a boron-doped liquid scintillator and a multi-detector directional spectrometer. As an outcome of these simulations, possible adaptations to these instruments are suggested with a view to improving the portability of the instrument.
\end{abstract}

Keywords: MCNP, dosimetry, directional neutron survey, neutron capture, critical review

\section{Introduction}

When estimating the health risk of exposure to neutron fields, the term Effective Dose can be used [1]. This provides a weighted sum of the absorbed dose across the human body. This non-isotropic quantity is not measurable.

Usually, ambient dose equivalent $H^{*}(10)$ is used to provide an operational quantity for area surveys. This considers the risk of a neutron field in an area, in a conservative manner, by being independent of the incident direction of the radiation field and approximating effective dose for the direction of incidence that has the greatest risk, i.e anteroPosterior (AP). In order to quantify the risk to a person exposed to the neutron field, personal dose equivalent is used. Personal dose meters are typically worn on the front of the torso and affects of shielding by the body can effect the readings. This reading is also considered a conservative estimate of the risk making the dose recorded likely to be under-estimated for predominantly postero-anterior (PA) exposure. In 2002 Barlett et al [2] highlighted a potential limitation of existing neutron survey techniques, due to lack of consideration to the direction of the field. In some organisations, neutron survey measurements are used for establishing work practices. Due to the conservative nature of the operational quantities, there is potential to over restrict these work practices. Furthermore, although effective dose is not recommended to be used for epidemiological studies, if such an instrument could estimate the effective dose within an area, such studies could only be improved. Research undertaken to assess the effectiveness of the measurement of these quantities in workplaces identified that both energy and direction of the neutron field should be considered [3]. Furthermore, from these studies it can be seen that ambient dose equivalent is not always a conservative estimate of the effective dose. This highlights the need for an instrument that can accurately measure energy and directional dependence of a neutron field.

\section{Existing Neutron Survey Techniques}

The method of detecting thermal neutrons within a range of hydrogenous moderators, known as a Bonner Sphere Spectrometer system (BSS), is a well established technique for neutron spectroscopy. As such, the response of this

\footnotetext{
*E-mail address: m.balmer@lancaster.ac.uk (M. J. I. Balmer)
} 


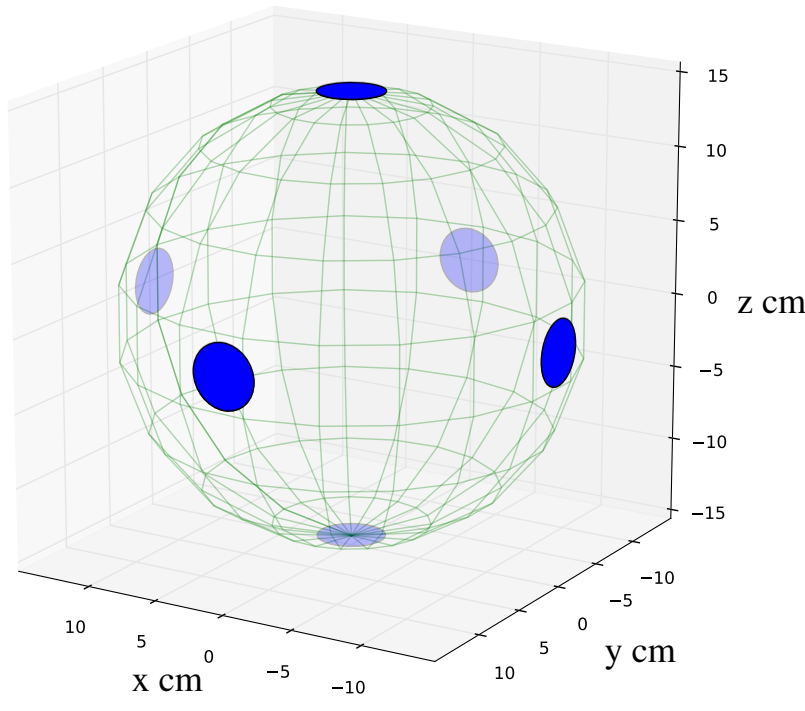

(a)

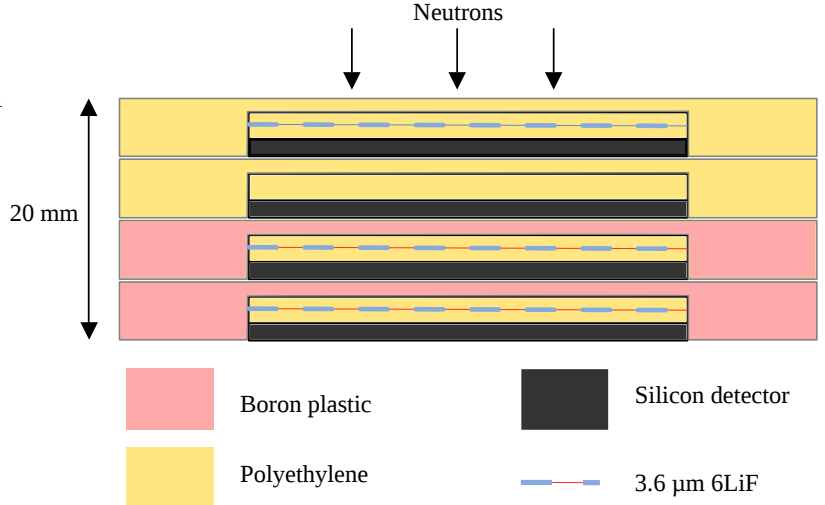

(b)

Figure 1: Detector developed by PTB as part of the EVIDOS campaign. (a) The 6 detectors are located around the perimeter of the $30 \mathrm{~cm}$ diameter polyethylene sphere. (b) Each detector consists of 4 sandwiched silicon detectors, each optimised for detection at different energy levels. Fig. replicated from [6].

instrument has been well characterised [4]. Combining this response with unfolding techniques, reasonably reliable neutron spectroscopy can be achieved. However, the time consuming nature and complexity of taking such field measurements makes it counterproductive. This technique also does not permit real-time neutron surveys to be achieved.

Existing neutron survey instruments measuring $H^{*}(10)$ which are commonly used, including the Leake Detector, NM2 and Studsvik instrument [3], are typically single detector instruments. Inferring the direction of the field using such instruments would present a tough challenge due to the single detector design of these instruments. However, such instruments have been in operation for many years and can be used as a basis for establishing the requirements of a practical directional neutron survey instrument that is portable. For daily area survey measurements to be taken in a workplace, an instrument needs to be portable and allow real time data capture to take place. It is anticipated it would be powered by batteries to allow for maximum portability of such an instrument.

With a portable battery powered directional neutron survey instrument, it is anticipated accurate data sets for work places could be obtained, and would have potential to improve work practices. This would also improve health studies on the effects of neutron radiation.

A broad range of neutron detection techniques have been employed to develop numerous instruments that could be used for directional neutron spectrometry. These instruments are reviewed and their required additional improvements, for use as a real-time portable directional neutron survey meter, are considered.

Perhaps the most comprehensive work undertaken to date in the field of directional neutron dosimetry is the 'Evaluation of Individual Dosimetry in Mixed Neutron and Photon Radiation Fields' (EVIDOS) project. The EU funded EVIDOS campaign was undertaken to attempt to understand the effectiveness of personal dosimetry instruments in workplace fields, considering both energy and direction dependence of the field. In this project, to enable a number of workplace fields to be characterised in a timely manner, two very different instruments were developed. The first was a telescope design directional neutron spectrometer [5], the second a directional spectrometer which could be likened to a collection of personal dose meters around a phantom [6].

\subsection{Multi-Detector Directional Spectrometer}

The instrument developed by Physikalisch-Technische Bundesanstalt (PTB), as part of the EVIDOS project consists of 6 neutron detectors located on the outer circumference of a polyethylene phantom (Fig. 1). In order to 
achieve high sensitivity detection with a flat response across the range of $0-20 \mathrm{MeV}$ poses a challenge in a single diode package. To acheive these goals, multi-element detectors consisting of 4 sandwiched silicon detectors within a single aluminium housing were used in this design. For detection at lower neutron energy levels, boron-doped plastic surrounds the ${ }^{6} \mathrm{LiF}$ coated silicon diode. For higher energy neutrons, a $30 \mu \mathrm{m}$ polyethylene layer is employed to allow for detection of charged particles produced by proton recoil events.

The response for each of the 24 detectors at different energies and directions presents a complex matrix for the response function of the instrument. This matrix was then used for unfolding the neutron spectrum. As part of the EVIDOS campaign this detector was successfully used to characterise a number of work place fields. This characterisation was verified in conjunction with BSS measurements.

By considering the dose rates experienced by each detector and calibrated data, it could perhaps be worthwhile to investigate if this data could train a neural network to predict the effective dose. Such a technique could be implemented on an FPGA and promote itself well to real-time operation. However with only 6 detectors it could prove difficult to achieve a good angular resolution with a neural network. A $30 \mathrm{~cm}$ polyethylene sphere is used in this design which has a mass of around $13.6 \mathrm{~kg}$. When coupled with digitising electronics this would present a challenge to make this device a portable instrument. For optimal results, prior information on the energy spectrum under investigation was required to aid the unfolding process [7]. This presents challenges when working in an unknown field where an instrument such as a Bonner Sphere System is not available. This restricts the instruments usage as a true real-time neutron dosimetry meter.

\subsection{Superheated Emulsion Detectors}

The 'directional telescope' developed for the EVIDOS project [5], presents a novel instrument using a superheated emulsion detector. The instrument consists of a $30 \mathrm{~cm}$ moderating sphere with a window subtending from the outer perimeter of the sphere through the detector located at the centre of the detection. When neutrons enter the detector, a superheated emulsion of dichlorotetrafluoroethane (R-114), bubbles form. These bubbles are then detected acoustically using peizo detectors. By controlling the temperature of this supherheated emulsion, different neutron detection thresholds can be set. The superheated detector is located within the centre of a $30 \mathrm{~cm}$ nylon- 6 sphere. This neutron moderator means that the only neutrons being detected should be those which have travelled the telescope window to the centre of the sphere. By rotating the sphere and controlling the temperature, energy and direction of the neutron field can be understood using a suitable unfolding technique.

Due to its time consuming data collection process, this instrument was not used for the EVIDOS campaign [7]. As a result of these limitations, the instrument does not have the same proven ability in an experimental capacity in a workplace field as the PTB spectrometer. When considering the practical implication of the design of a neutron survey meter, the portability is a strong influencing factor on the design. Power drain for the complex temperature cycling and detector rotation would restrict this instruments operating time using batteries as well as impacting on the mass of the instrument.

Using optical readout of bubbles instead of acoustic readout presents another interesting option for directional neutron dosimetry [8]. The instrument consists of a beam of light crossing a superheated emulsion (Fig. 2). As this beam of light crosses the detector, the bubbles formed due to detection of neutrons cause scattering of light, can be detected in real time, using silicon photo diodes. This information on the bubble distribution could be used to estimate the direction and energy of the neutron field entering the detector. The development of such techniques within a practical detector are still in their early stages and issues such as life span of these detectors are still under investigation [9].

\subsection{Single Bonner Sphere Spectrometers}

Several instruments $[10,11]$ have been developed incorporating multiple detectors within a single moderating sphere, thus eliminating the need for multiple BSS instruments when characterising a neutron field. Such instruments were not specifically designed for the purpose of directional neutron dosimetry, but could be adapted to be used in such an application. However the design of many of these instruments relies on ${ }^{3} \mathrm{He}$, which has gone into short supply in the last ten years following increased concern over nuclear security, following the events of September 2001 [12]. Work has begun to attempt to develop these designs with alternatives to the hard to source ${ }^{3} \mathrm{He}$ [13], however many of these methods of detection remain in their early stages of development. 


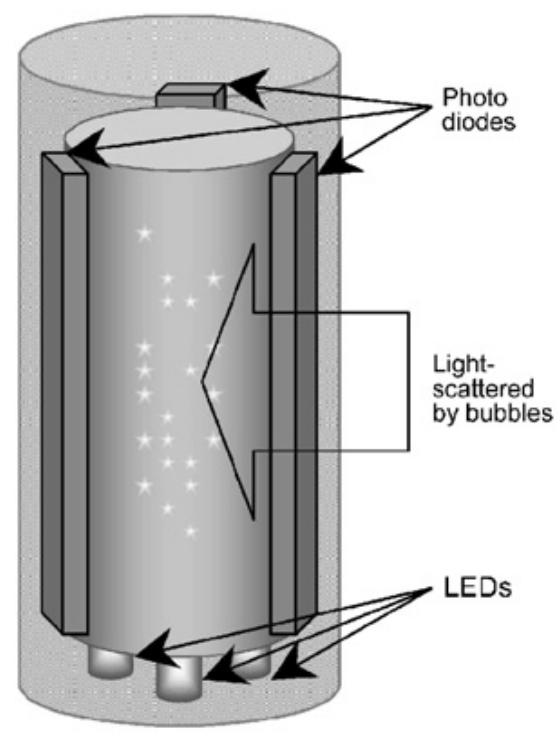

Figure 2: An example of a Superheated Emulsion Detector using optical detection of the bubbles. [8].

A passive spectrometer for workplace monitoring with a quasi-isotropic response to neutrons, from thermal to fast energies, was designed by Gómes Ros et al [14]. Earlier developments [15] of this instrument used ${ }^{6} \mathrm{LiF}$ and ${ }^{7} \mathrm{LiF}$ thermoluminescent detectors embedded within a single polyethylene sphere. These detectors were replaced by Dysprosium activation foils to improve photon insensitivity. Following experimentation with a $14 \mathrm{MeV}$ neutron beam, the resulting Dy-foils-based Single Sphere Spectrometer (Dy-SSS) showed promising results with an overall uncertainty of the spectrometer response matrix of $\pm 3 \%$. The goal of such instruments is to eliminate any directional bias in the instrument, by averaging the response readings along each axis of the instrument. With careful characterisation such an instrument could perhaps be used for directional dosimetry. However the response matrix such a complex array of instruments would pose a tough challenge to permit real-time unfolding of the neutron spectrum.

\subsection{Boron-Doped Spherical Scintillator Detector}

A theoretical design of a directional neutron survey meter was carried out by Taylor in 2010 [16]. This instrument is a boron-doped spherical scintillator of $20.32 \mathrm{~cm}$ diameter interrogated by multiple photomultiplier tubes (PMT). The boron-doped scintillator allows for neutron detection from thermal up to energy ranges of the order of tens of $\mathrm{MeV}$ (depending on dimensions of the scintillator). When a neutron enters the scintillator, the elastic collisions causing proton recoil events allow high energy neutrons to be detected. This proton recoil event can then be detected through a scintillation event as a result of this elastic collision. The boron loading of the BC-523A scintillator allows for detection of thermal neutrons through the $\alpha$ particle emitted as a result of the neutron capture. The capture distribution of scintillation events within the detector can then be analysed to provide an estimate of effective dose.

Through Monte-Carlo modelling, Taylor obtained a series of capture distributions for different energies and locations of mono-energetic neutron sources. An artificial neural network was then trained with these capture distributions to learn the pattern of the distribution for a given energy. With preliminary training of the neural network, promising results were achieved when the model was bench marked with a ${ }^{252} \mathrm{Cf}$ source. However in this proof of concept localising the scintillation event within the detector remains unproved.

Localising the scintillation event within the detector will present a challenge. It is thought that using an array of PMTs coupled to a suitable digital signal processing algorithm, the scintillation event in the detector can be localised. Although the $2 \times 2 \times 2 \mathrm{~cm}^{3}$ voxels used for the simulation present what could be considered a practical size of voxel for a modern multi-channel analyser, it is unknown if this resolution of localisation can be achieved.

Training the artificial neural network will present many challenges. Training with only modelled results could lead to possible inaccuracies. However to best train the network, a large number of energy levels and directions would 


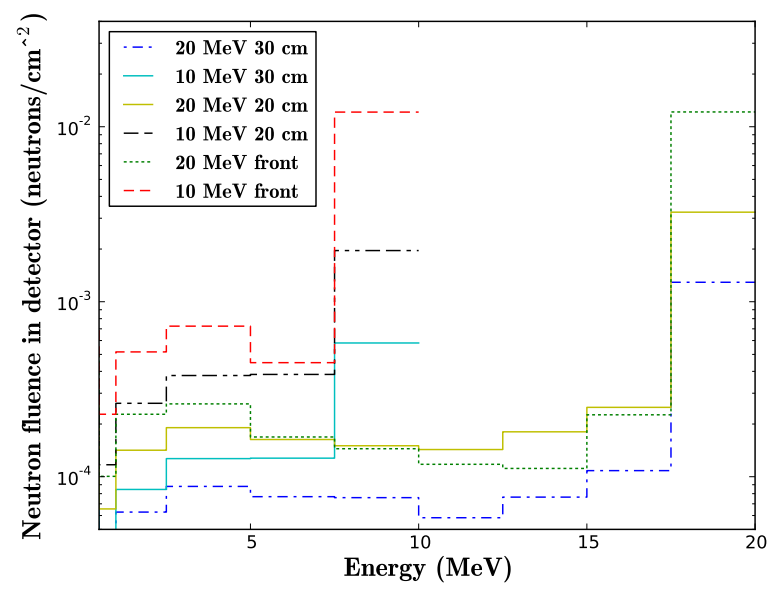

Figure 3: Investigating reducing the size of polyethylene moderator shows that by reducing the diameter of the sphere to $20 \mathrm{~cm}$, an upper limit of $10 \mathrm{MeV}$ is set to achieve a similar performance of a $30 \mathrm{~cm}$ diameter sphere at $20 \mathrm{MeV}$.

be needed to be experimentally obtained, doing this through practical experiments could be prohibitively expensive and/or time consuming.

The flash point of the BC-523A scintillator is a potential cause for concern from a practical perspective of developing such an instrument. A more practical alternative for similar performance is the EJ-309B5 scintillator, which has a much safer flash point above one hundred degrees centigrade [17]. In such boron-loaded scintillators, the $\alpha$ particle emitted during boron capture events, causes a strong quenching of the scintillation event [18]. Within a scintillator of the size proposed, this could perhaps lead to difficulties detecting such low amplitude pulses. Promising recent developments in the field of crystal scintillators makes $\mathrm{Cs}_{2} \mathrm{LiYCl}_{6}: \mathrm{Ce}$ (CLYC) a scintillator worthy of consideration for use in this instrument [19].

\section{Investigating Possible Improvements To Existing Designs}

\subsection{Simulating Different Moderator Sizes}

When considering the upper energy detection range of a neutron detector, a trade off has to be considered between neutron moderation and portability. Typically designs utilise a $30 \mathrm{~cm}$ polyethylene phantom to moderate the incoming neutrons and such a phantom has a mass of $13.6 \mathrm{~kg}$. By reducing the size of this phantom, the difference in fluence between the detectors at the front and back of the phantom will be lessened. This reduction in fluence differential will impact on the directional resolution of the detector. However a reduction to a $20 \mathrm{~cm}$ sphere equates to a mass of $4 \mathrm{~kg}$, an attractive mass reduction. In order to investigate the relationship between this upper energy limit and sphere mass, a typical detector was modelled using a Monte-Carlo radiation transport code package, MCNP v5.0.

A $30 \mathrm{~cm}$ polyethylene sphere (density $\left.0.96 \mathrm{~g} / \mathrm{cm}^{3}\right)$ was modelled with two discs $(2.5 \mathrm{~cm}$ diameter, $1 \mathrm{~mm}$ thickness) embedded diametrically opposite each other at a depth of $1 \mathrm{~cm}$ within the sphere. On the front face of the moderator detector 1 was closest to the neutron source. Within the back of the moderator, furthest from the source, detector 2 was located along the same axis. The detector was irradiated by mono-energetic, mono-directional neutrons. Sphere diameters of $20 \mathrm{~cm}$ and $30 \mathrm{~cm}$ were modelled, each being sequentially radiated by a $10 \mathrm{MeV}$ and $20 \mathrm{MeV}$ neutron source. The fluence within each detector was recorded in discrete energy bins and the fluence between the front and back detector was compared. The results can be seen in Fig. 3.

By reducing the sphere to $20 \mathrm{~cm}$ diameter, an improvement in fluence difference to that at $20 \mathrm{MeV}$ with a $20 \mathrm{~cm}$ diameter sphere can be achieved. However this difference in fluence is still smaller than that of a $30 \mathrm{~cm}$ diameter sphere at $20 \mathrm{MeV}$. This reduction in fluence difference could impact on the performance of the unfolding codes. 


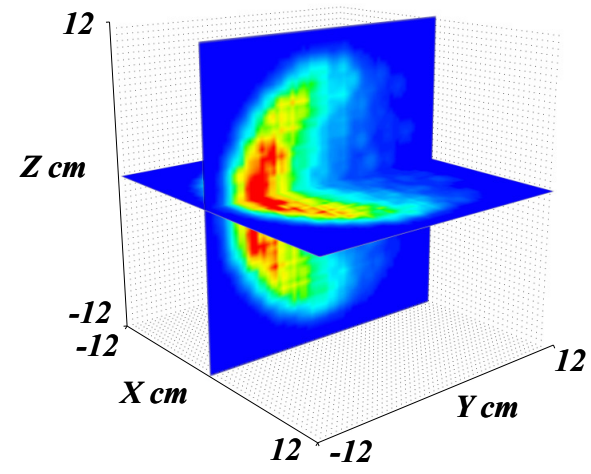

(a)

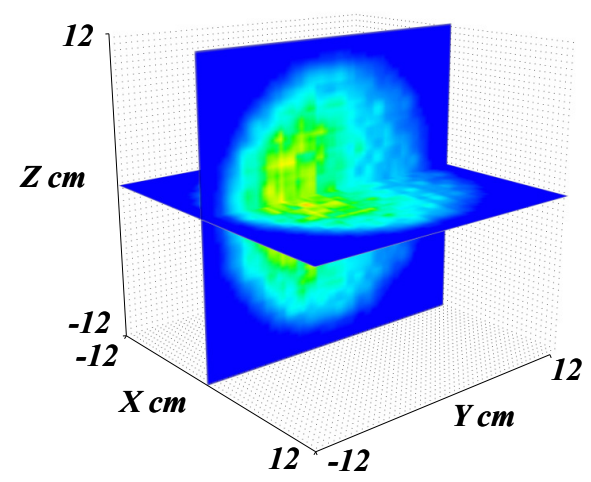

(c)

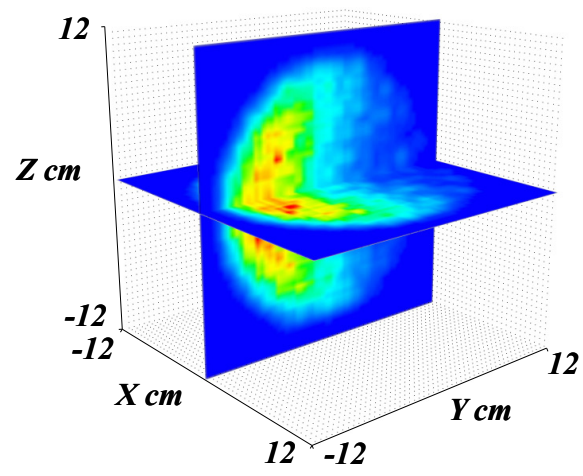

(b)

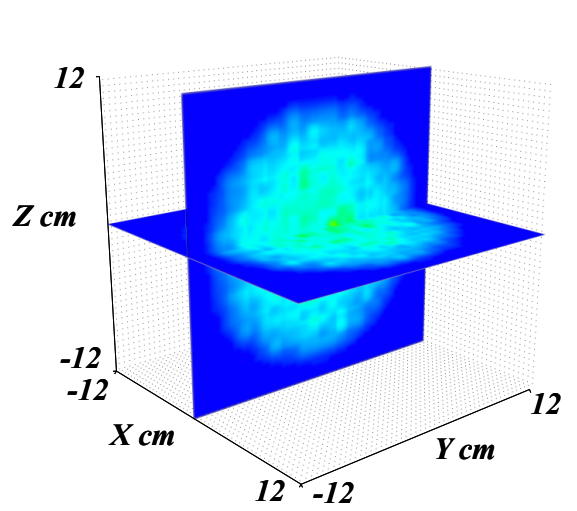

Counts per Voxel

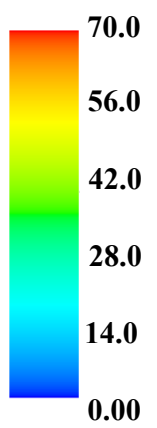

(d)

Figure 4: Distribution of neutron captures within a $20.32 \mathrm{~cm}$ diameter boron loaded BC-523A scintillator exposed to four different mono-energetic neutron energies, (a) $10 \mathrm{keV}$, (b) $100 \mathrm{keV}$, (c) $1 \mathrm{MeV}$ and (d) $10 \mathrm{MeV}$

\subsection{Neutron Capture Distribution Within A Scintillator}

The artificial neural network used by Taylor [16] for the scintillator detector relies on there being directional information within the pattern of the capture distribution. The detector was modelled in MCNP with a BC-523A scintillator, to investigate these distributions at different energies. The detector was irradiated with AP radiation from a mono-energetic neutron source, for four different energies over the range of $10 \mathrm{keV}$ to $10 \mathrm{MeV}$. Using the PTRAC output, the coordinates of $10^{5}$ capture events were binned into $1 \mathrm{~cm}^{3}$ voxels. Using the Mayavi [20] package, the capture distribution of the events within the detector are shown in Fig. 4.

At $10 \mathrm{keV}$ it can be seen that there is a strong directional indication of direction from the distribution of captures. However at $1 \mathrm{MeV}$ it can be seen that there is a weaker indication of capture distribution, and as $10 \mathrm{MeV}$ is approached, it is not possible to visually distinguish the direction of the source.

By increasing the size of the scintillator to $30 \mathrm{~cm}$ diameter and considering $10^{6}$ neutron capture events, the detector was remodelled. By increasing the scintillator to this size it would increase the mass from $4.8 \mathrm{~kg}$ to $12.9 \mathrm{~kg}$. It can be seen in Fig. 5 that the increase in size of the scintillator has not improved the directional information, within the pattern of the capture distribution. For this significant increase in mass of the scintillator, no improvement to the upper energy limit of detection is achieved.

\section{Discussion}

Existing neutron dosimetry techniques have been reviewed within this paper and their suitability to application within a portable neutron dose meter has been reviewed. When considering the practical aspects using existing 


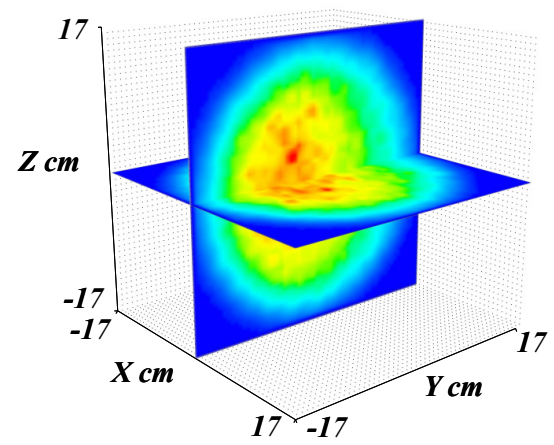

Counts per Voxel

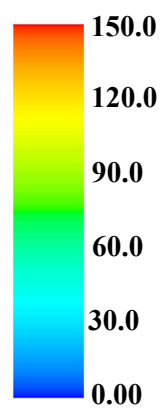

Figure 5: Distribution of neutron captures with a $30 \mathrm{~cm}$ diameter boron-loaded BC-523A scintillator exposed to a mono-energetic beam of $10 \mathrm{MeV}$

instruments for portable applications, the heavy mass of large moderators restricts their usage. It has been shown that by reducing the upper energy range of the detector, a much lighter mass of moderator can be used with an estimated upper limit of $10 \mathrm{MeV}$ for detection. The detector developed by PTB design could be reduced in size and a much lighter portable instrument could be realised. However the pre-information required for the unfolding restricts the instruments usage as a true real-time instrument.

Although only theoretical, the design proposed by Taylor presents an attractive option for a portable digitised neutron dosimeter. This instrument, holds promise to be a lightweight instruments which could be coupled with a portable digital analyser. Using such digital detection techniques could offer a large degree of versatility through software. With a digital architecture $(\mathrm{n}, \gamma)$ discrimination techniques could be implemented onto an FPGA multi-channel analyser. Furthermore, with careful selection of scintillator, it could be possible to implement gamma dosimetry within the same hardware. Also as has been highlighted within the paper, the proposed BC-523A scintillator not only limits the upper range of detection, but also outputs a very low amplitude pulse of light as a result of a capture event.

Being able to reliably detect these pulses of light, as a result of the neutron capture, presents remains an unproven concept. The resolution and reliability of this capture localisation will have a big impact on the efficiency and accuracy of the detector.

\section{Acknowledgements}

The authors would like to acknowledge the funding support from EPSRC and National Physical Laboratory, Teddington, UK. We also acknowledge the help and advise of Prof. Malcolm Joyce at Lancaster University and Dr. Nigel Hawkes at National Physical Laboratory.

\section{References}

[1] ICRP, The 2007 recommendations of the international commission on radiological protection, Annals of the ICRP 37 (2-4) (2007) $49-79$.

[2] D. Bartlett, P. Drake, F. d'Errico, M. Luszik-Bhadra, M. Matzke, R. Tanner, The importance of the direction distribution of neutron fluence, and methods of determination, Nuclear Instruments and Methods in Physics Research Section A: Accelerators, Spectrometers, Detectors and Associated Equipment 476 (1-2) (2002) 386 - 394.

[3] R. Tanner, C. Molinos, N. Roberts, D. Bartlett, L. Hager, G. Taylor, D. Thomas, Practical implications of neutron survey instrument performance, Tech. Rep. HPA-RPD-016, Health Protection Agency, Chilton, UK (2006).

[4] D. J. Thomas, A. V. Alevra, Bonner sphere spectrometersa critical review, Nuclear Instruments and Methods in Physics Research Section A: Accelerators, Spectrometers, Detectors and Associated Equipment 476 (1) (2002) 1220.

[5] F. d'Errico, V. Giusti, M. Reginatto, B. Wiegel, A telescope-design directional neutron spectrometer, Radiation Protection Dosimetry 110 (14) (2004) 533-537.

[6] M. Luszik-Bhadra, F. d'Errico, O. Hecker, M. Matzke, A wide-range direction neutron spectrometer, Nuclear Instruments and Methods in Physics Research Section A: Accelerators, Spectrometers, Detectors and Associated Equipment 476 (1) (2002) 291297. 
[7] F. d'Errico, D. Bartlett, T. Bolognese-Milsztajn, M. Boschung, M. Coeck, G. Curzio, A. Fiechtner, J.-E. Kyllonen, V. Lacoste, L. Lindborg, M. Luszik-Bhadra, M. Reginatto, H. Schuhmacher, R. Tanner, F. Vanhavere, Evaluation of individual dosimetry in mixed neutron and photon radiation fields (EVIDOS). part i: scope and methods of the project, Radiation Protection Dosimetry 125 (1-4) (2006) $275-280$.

[8] F. d'Errico, A. Di Fulvio, M. Maryaski, S. Selici, M. Torrigiani, Optical readout of superheated emulsions, Radiation Measurements 43 (2-6) (2008) 432-436.

[9] F. d'Errico, A. Di Fulvio, Superheated emulsions for the detection of special nuclear material, Radiation Measurements 46 (12) (2011) 1690-1693.

[10] D. T. Bartlett, R. J. Tanner, D. G. Jones, A new design of neutron dose equivalent survey instrument, Radiation protection dosimetry 74 (4) (1997) 267271

[11] S. D. Monk, M. J. Joyce, Z. Jarrah, D. King, M. Oppenheim, A portable energy-sensitive cosmic neutron detection instrument, Review of Scientific Instruments 79 (2) (2008) 023301

[12] P. Peerani, A. Tomanin, S. Pozzi, J. Dolan, E. Miller, M. Flaska, M. Battaglieri, R. De Vita, L. Ficini, G. Ottonello, G. Ricco, G. Dermody, C. Giles, Testing on novel neutron detectors as alternative to 3 He for security applications, Nuclear Instruments and Methods in Physics Research Section A: Accelerators, Spectrometers, Detectors and Associated Equipment 696 (2012) 110-120.

[13] M. Selwood, S. Monk, Development of a portable isotropic neutron spectrometer, Applied Radiation and Isotopes 70 (7) (2012) $1145-1149$.

[14] J. Gómez-Ros, R. Bedogni, I. Palermo, A. Esposito, A. Delgado, M. Angelone, M. Pillon, Design and validation of a photon insensitive multidetector neutron spectrometer based on dysprosium activation foils, Radiation Measurements 46 (12) (2011) $1712-1715$.

[15] J. Gómez-Ros, R. Bedogni, M. Moraleda, A. Delgado, A. Romero, A. Esposito, A multi-detector neutron spectrometer with nearly isotropic response for environmental and workplace monitoring, Nuclear Instruments and Methods in Physics Research Section A: Accelerators, Spectrometers, Detectors and Associated Equipment 613 (1) (2010) 127-133.

[16] G. C. Taylor, Design of a direction-dependent neutron dosimeter, Radiation Measurements 45 (10) (2010) 1301-1304.

[17] J. Iwanowska, L. Swiderski, M. Moszynski, Liquid scintillators and composites in fast neutron detection, Journal of Instrumentation 7 (04) (2012) C04004-C04004.

[18] L. Swiderski, M. Moszynski, D. Wolski, T. Batsch, A. Nassalski, A. Syntfeld-Kazuch, T. Szczesniak, F. Kniest, M. R. Kusner, G. Pausch, J. Stein, W. Klamra, Boron-10 loaded BC523A liquid scintillator for neutron detection in the border monitoring, IEEE Transactions on Nuclear Science 55 (6) (2008) 3710-3716.

[19] J. Glodo, R. Hawrami, K. Shah, Development of Cs2LiYCl6 scintillator, Journal of Crystal Growth.

[20] P. Ramachandran, G. Varoquaux, Mayavi: 3D Visualization of Scientific Data, Computing in Science \& Engineering 13 (2) (2011) $40-51$. 